\title{
The Anisotropic Lagrangian Averaged Euler and Navier-Stokes Equations
}

\author{
JERROLD E. MARSDEN \& STEVE SHKOLLER \\ Dedicated to Stuart Antman on the occasion of his 60th birthday
}

Communicated by S. MULLLR

\begin{abstract}
The purpose of this paper is twofold. First, we give a derivation of the Lagrangian averaged Euler (LAE- $\alpha$ ) and Navier-Stokes (LANS- $\alpha$ ) equations. This theory involves a spatial scale $\alpha$ and the equations are designed to accurately capture the dynamics of the Euler and Navier-Stokes equations at length scales larger than $\alpha$, while averaging the motion at scales smaller than $\alpha$. The derivation involves an averaging procedure that combines ideas from both the material (Lagrangian) and spatial (Eulerian) viewpoints. This framework allows the use of a variant of G. I. Taylor's "frozen turbulence" hypothesis as the foundation for the model equations; more precisely, the derivation is based on the strong physical assumption that fluctutations are frozen into the mean flow. In this article, we use this hypothesis to derive the averaged Lagrangian for the theory, and all the terms up to and including order $\alpha^{2}$ are accounted for.

The equations come in both an isotropic and anisotropic version. The anisotropic equations are a coupled system of PDEs (partial differential equations) for the mean velocity field and the Lagrangian covariance tensor. In earlier works by FOIAS, HOLM \& TITI [10], and ourselves [16], an analysis of the isotropic equations has been given. In the second part of this paper, we establish local in time well-posedness of the anisotropic LANS- $\alpha$ equations using quasilinear PDE type methods.
\end{abstract}

\section{Introduction}

Given a fluid domain $\Omega \subset \mathbb{R}^{3}$, the velocity-pressure representation of the incompressible Navier-Stokes (NS) equations is given by

$$
\left.\begin{array}{r}
\partial_{t} u+(u \cdot \nabla) u=-\operatorname{grad} p+v \Delta u, \\
\operatorname{div} u=0,
\end{array}\right\} \begin{gathered}
\text { on } \Omega \times(0, T), \\
\left.u(t, x)\right|_{t=0}=u_{0}(x), \quad u(t, x)=0 \text { on } \partial \Omega, t \in[0, T) ;
\end{gathered}
$$


here, $v>0$ is the kinematic viscosity, $u(t, x)$ is the spatial velocity vector, and $p(t, x)$ is the scalar pressure field, which is determined up to a constant from the velocity field $u$. It is widely accepted that (1) contains all of turbulence (see, for example, page 1 of FRISCH [11]); nevertheless, even with knowledge of the mathematical model, the problem of turbulence remains one of the last great unsolved problems of physics. The convective nonlinearity $(\nabla \cdot u) u$ sends energy from the large spatial scales into smaller and smaller scales until the energy reaches the Kolmogorov dissipation scale, at which it is annihilated by the linear dissipation mechanism $v \Delta u$. This energy cascade reaps computational havoc; in order to resolve a numerical simulation of (1), enough grid points or Fourier modes must be used so that the approximation captures all of the spatial scales down to the Kolmogorov scale. For turbulent flows, such resolution requirements remain prohibitively expensive.

Reynolds Averaging. In turbulent regimes, it is reasonable to propose a statistical theory of turbulence. For this purpose, it is often supposed that the velocity field of the fluid is a random variable which may be represented by the Reynolds decomposition

$$
u(t, x)=U(t, x)+u^{\prime}(t, x)
$$

where $u^{\prime}(t, x)$ denotes a random variable with mean value zero, $\overline{u^{\prime}}=0$, and that it is only knowledge of the averaged value of velocity $U(t, x)$ that will be important for applications. A mathematical model for the evolution of the averaged velocity $U$ requires the derivation of an averaged Navier-Stokes equation, which would greatly facilitate our understanding of the NS equation (1). The classical approach for deriving such an averaged Navier-Stokes model, is to substitute the decomposition (2) into the NS equation (1) and then average. This procedure yields the Reynolds averaged Navier-Stokes (RANS) equations, which are given by

$$
\partial_{t} U+(U \cdot \nabla) U+\operatorname{Div} \overline{u^{\prime} \otimes u^{\prime}}=-\operatorname{grad} P+v \Delta U .
$$

The tensor $\overline{u^{\prime} \otimes u^{\prime}}$ is called the Reynolds stress and leads to the so-called turbulent closure problem, namely that of expressing the Reynolds stress in terms of the mean velocity $U$ so that $U$ has well-defined dynamics.

Classically, it is assumed that the Reynolds stress tensor depends only on the gradient of the mean velocity field $\nabla U$ and that the mapping $\nabla U \mapsto \overline{u^{\prime} \otimes u^{\prime}}$ is Galilean-invariant; these assumptions yield the result

$$
\overline{u^{\prime} \otimes u^{\prime}}=v_{E}(t, x, \operatorname{Def} U) \cdot \operatorname{Def} U,
$$

where the (rate of) deformation or strain tensor Def $U$ is given by

$$
\text { Def } U=\frac{1}{2}\left[\nabla U+(\nabla U)^{T}\right],
$$

and $v_{E}$ is the eddy viscosity which in general may depend on $t, x$ and Def $U$ in a nonlinear fashion.

As a result of such an averaging approach, artificial viscosity is added into the system to remove energy which is contained in the small scales at which $u^{\prime}$ resides, and there is still a need to guess the functional form of the eddy viscosity $v_{E}$. 
The Need for an Alternative Approach. The LANS- $\alpha$ equations ${ }^{1}$, in their isotropic and anisotropic versions, directly deal with this problem and, besides being theoretically interesting, have proved to be computationally very attractive. The inviscid form of the isotropic version of these equations on $\mathbb{R}^{n}$ can be traced back to [13, 14] (and on compact Riemannian manifolds to [22]), but there have been numerous extensions made to both the viscous and bounded domain settings, and we refer the reader to [16] for the history of this subject and numerous references. The anisotropic equations appeared in HOLM [12] under the name of LMM equations-see Remark 1 below.

While the (isotropic) equations have been extensively studied, a correct derivation that accounts for all the terms up through order $\alpha^{2}$ has been missing in the literature. Our first goal in this paper is to provide a precise set of assumptions that yield the averaged action principle from which these equations (both the isotropic and anisotropic cases) arise. Our second goal is to give a local existence and uniqueness theorem for the anisotropic case on regions without boundary. The case of turbulent channel flow and flow on general bounded domains is treated in [6] and [7].

The general technique for the derivation is to perform averaging at the level of the action principle. Such an approach is common in other areas where the variational structure of the problem is perhaps more transparent. Examples are in nonlinear waves and the derivation of envelope equations, notably in WHITHAM [26] and [9]. It also occurs in the Physics literature, as in, for example, [24]. Thus, while the general idea for such an approach is not new, its application to the derivation of the averaged fluid equations is relatively recent.

Generalizing the approach used in [16] for the isotropic case, we propose a technique for dealing with the averaging of (1) which uses a modern version of TAYLOR's Frozen Turbulence Hypothesis (see [25]) to realize the turbulence closure. In our approach, we replace the fundamental additive Reynolds decomposition (2) by a decomposition of volume-preserving flows, and perform an ensemble averaging of the energy of the fluid, rather than averaging the Navier-Stokes equations themselves. The procedure makes fundamental use of the interplay between the Eulerian and Lagrangian representations of the fluid ${ }^{2}$. Our method is also related to

1 These equations have also been referred to as the viscous Camassa-Holm equations by Foias, Holm, Titi and their collaborators. Other names that have been used in the literature for the LANS- $\alpha$ equations are either the Navier-Stokes- $\alpha$ or $\alpha$-Navier-Stokes equations.

2 As Stuart Antman has often pointed out, the Eulerian-Lagrangian historical attribution is not entirely correct, but it is in common use, so we will follow it. Lagrange's insight into both fluid mechanics and rigid body mechanics in his Mechanique Analytique is quite remarkable. For example, he derives (in volume II) the rigid-body equations for a general quadratic Lagrangian using what we would call today Euler-Poincaré reduction from material to body representation and he implicitly realized that the same process is at work in fluid dynamics. He also discovered that the flow of the Euler-Lagrange equations is symplectic; see [15] for further information. A key point in the derivation of the averaged fluid equations is that we do not simply average the spatial equations. Rather, paying careful attention to the material-spatial representations and the variational structure of each, gives key insights into the whole averaging process and is fundamental to our approach. 
the method of optimal prediction (see [5]), since both theories make use of invariant measures to average over the uncertainty in specifying initial data.

In carrying this out, and as already indicated, averaged models in two types of flow regimes are obtained. The first regime, common in period-box numerical simulations, consists of isotropic turbulence in which the covariance tensor is assumed to be a multiple of the identity tensor. The second regime is for anisotropic turbulence, appropriate for shear flows and flows over obstacles, wherein the covariance tensor plays a prominent role in the mechanics of the problem.

We shall not discuss the literature for numerical simulations of isotropic turbulence in this paper, but refer to [18] for a survey and further references.

The LANS- $\alpha$ Equations. The isotropic Lagrangian averaged Navier-Stokes (LANS$\alpha$ ) equations on domains without boundary, such as in a periodic box, are given by

$$
\begin{gathered}
\partial_{t} u+(u \cdot \nabla) u+\operatorname{div} \tau^{\alpha}=-\operatorname{grad} p+v \Delta u, \\
\operatorname{div} u=0,\left.\quad u(t, x)\right|_{t=0}=u_{0}(x),
\end{gathered}
$$

where $\alpha>0$ is a small spatial scale, below which the fluid motion of the NavierStokes equations is averaged, the subgrid or Reynolds stress $\tau^{\alpha}$ is given by

$$
\tau^{\alpha}=\alpha^{2}\left(1-\alpha^{2} \Delta\right)^{-1}[\operatorname{Def}(u) \cdot \operatorname{Rot}(u)],
$$

where $\operatorname{Rot}(u)=\left(\nabla u-\nabla u^{T}\right) / 2$ is the antisymmetric part of the velocity gradient, and $\left(1-\alpha^{2} \Delta\right)$ is the Helmholtz operator.

The tensor $\tau^{\alpha}$ is antisymmetric, but we can also write, if desired, $\operatorname{div} \tau^{\alpha}=\operatorname{div} \tilde{\tau}^{\alpha}$ where $\tilde{\tau}^{\alpha}$ is a symmetric tensor; see [18] for the explicit expression.

This form of the isotropic equations shows how the original Navier-Stokes equations are modified by the subgrid stress term $\tau^{\alpha}$. The effect of the subgrid stress $\tau^{\alpha}$ is to provide an a priori estimate for $u$ in $L^{\infty}\left(0, T ;\left[H^{1}(\Omega)\right]^{3}\right)$ for $T>0$, from which global existence and uniqueness of smooth solutions easily follows.

The original form of the isotropic LANS- $\alpha$ equations on a periodic box, written as

$$
\begin{array}{r}
\partial_{t}\left(1-\alpha^{2} \Delta\right) u+(u \cdot \nabla)\left(1-\alpha^{2} \Delta\right) u+[\nabla u]^{T} \cdot\left(1-\alpha^{2} \Delta\right) u \\
=-\operatorname{grad} p+v \Delta\left(1-\alpha^{2} \Delta\right) u, \quad \operatorname{div} u=0,
\end{array}
$$

was first introduced in CHEN et al. [3], and the first global existence theorem for these equations was given in the work of FOIAS, HOLM \& TITI [10].

As noted in [23] and [16], on a bounded domain, the viscous term must be replaced with $v\left(1-\alpha^{2} \Delta\right) P \Delta u$, where $P$ is the Leray projector onto divergence-free vector fields (see Remark 1 below). This change yields global well-posedness for the isotropic LANS- $\alpha$ equations on open bounded subsets $\Omega \subset \mathbb{R}^{3}$ (see [16] for a proof, as well as a discussion of the viscous term on bounded domains).

In this article, our PDE analysis will focus on the anisotropic Lagrangian averaged Navier-Stokes (LANS- $\alpha$ ) equations. The anisotropic LANS- $\alpha$ equations are 
a coupled system of PDEs for the mean fluid velocity $u$ and the covariance matrix $F$. The covariance tensor is a symmetric two-tensor with both indices up. The anisotropic equations are given on $\Omega \times(0, T)$ by

$$
\begin{aligned}
\left(1-\alpha^{2} C\right)\left(\frac{D u}{D t}-v \mathbb{P} C u\right) & =-\operatorname{grad} p, \\
\operatorname{div} u(t, x) & =0, \\
\partial_{t} F+\nabla F \cdot u & =\nabla u \cdot F+[\nabla u \cdot F]^{T}, \\
u(0, x)=u_{0}(x), F(0, x) & =F_{0}(x) \geqq 0,
\end{aligned}
$$

where $D / D t:=\partial_{t}+(\nabla \cdot u)$ denotes the total time derivative, and

$$
C u=\operatorname{div}[\nabla u \cdot F],
$$

or in components $(C u)^{i}=\partial_{x^{k}}\left(F^{j k} \partial_{x^{j}} u^{i}\right)$, where the Einstein summation convention is used. Let $n$ denote the unit normal to $\partial \Omega$. While our derivation in Section 3 uniquely determines the inviscid $(v=0)$ equations, there is some choice in the viscous version (see also Remark 1 below). Namely, the operator $\mathbb{P}$ is a projection onto divergence-free vector fields and can be chosen to be either the classical Leray projector $P: L^{2}(\Omega) \rightarrow\left\{v \in L^{2}(\Omega) \mid \operatorname{div} v=0, v \cdot n=0\right.$ on $\left.\partial \Omega\right\}$, or the Stokes projector $\mathcal{P}_{F}^{\alpha}:=\left[P\left(1-\alpha^{2}\right) C\right]^{-1}\left(1-\alpha^{2} C\right)$ (defined in detail below in Definition 2 for the case where $\Omega=\mathbb{T}^{3}$ ). These projectors differ only in the boundary conditions which they assign to the divergence-free vector field.

On a bounded domain, we use the no-slip boundary conditions for $u$ given by

$$
u=0 \quad \text { on } \partial \Omega .
$$

For the transport equation (6c), written in components as $\partial_{t} F^{i j}+\partial_{x^{k}} F^{i j} u^{k}=$ $\partial_{x^{k}} u^{i} F^{k j}+\partial_{x^{k}} u^{j} F^{k i}$, we have the boundary condition

$$
F=0 \quad \text { on } \partial \Omega \text {. }
$$

The condition (9) is not surprising since $F$ is the average of the square of the fluctuations, and fluctuations must vanish along the boundary (since we are asking the turbulent velocity field to vanish along the boundary). Because of the condition (9), it is important to use the projector $\mathbb{P}=\mathcal{P}_{F}^{\alpha}$ on bounded domains; however, for domains without a boundary, such as a periodic box, $\mathbb{P}=P$ is a natural and simple choice.

The following basic energy law holds for sufficiently smooth solutions:

$$
\begin{aligned}
\frac{1}{2} \frac{d}{d t} \int_{\Omega}\left[|u|^{2}\right. & \left.+\alpha^{2} \operatorname{Trace}(\nabla u \cdot F \cdot \nabla u)\right] d x \\
& =-v \int_{\Omega}\left[\operatorname{Trace}(\nabla u \cdot F \cdot \nabla u)+\alpha^{2}|\mathbb{P} C u|^{2}\right] d x .
\end{aligned}
$$

Remark 1. For the case where $\mathbb{P}=\mathcal{P}_{F}^{\alpha}$, the equations (6) have appeared under the name of Lagrangian Mean Motion (LMM) equations as equations (16) in HoLM [12]. These equations essentially agree with our equations: solutions to both systems of equations produce the same velocity field but may yield different pressure functions. 
Remark 2. There is an intriguing connection between the isotropic Lagrangian averaged models of turbulence and certain models of non-Newtonian fluids, as well as certain numerical methods for solving the Euler and Navier-Stokes equations.

In particular, the Lagrangian averaged Euler (LAE- $\alpha$ ) equations are mathematically identical to the inviscid form of second-grade non-Newtonian fluids (see [21]) for which there is an extensive mathematical literature; however, the viscous term that is present in the second-grade fluids model is weaker than the viscosity in the LANS- $\alpha$ equations, acting more like damping than dissipation. Furthermore, the parameter $\alpha$ in that theory measures the elastic response of the fluid, whereas in our theory, $\alpha$ represents a spatial filtering scale.

It also happens that the LAE- $\alpha$ equations coincide exactly with the well-known vortex-blob algorithm for a certain choice of blob (smoothing) function. See [19] for a discussion of this topic, as well as for global well-posedness results for weak solutions in two dimensions, and convergence estimates of the vortex algorithm to solutions of the Euler equations in very weak topologies.

It may very well be the case that certain models of non-Newtonian fluids exist which coincide (or are related to) the anisotropic LANS- $\alpha$ equations. As far as vortex methods are concerned, we conjecture that the anisotropic equations are strongly related to the elliptical vortex-blob methods introduced in [17].

\section{The variational principle}

The Variational Approach to the Euler Equations. For $s>5 / 2$, let

$$
\begin{aligned}
\mathcal{D}_{\mu}^{s}=\{\eta: \Omega & \rightarrow \Omega \mid \eta \text { is bijective, } \eta(\partial \Omega) \subset \partial \Omega, \\
& \left.\eta \in H^{s}(\Omega, \Omega), \eta^{-1} \in H^{s}(\Omega, \Omega), \operatorname{Det}(D \eta)=1\right\} .
\end{aligned}
$$

This is the group of volume-preserving diffeomorphisms of the fluid container $\Omega$ of $H^{s}$-class regularity. It is well known (see [2] and [8]) that $\mathcal{D}_{\mu}^{s}$ is a smooth differentiable manifold, a topological group with right multiplication being smooth, and is the configuration space in incompressible hydrodynamics, since the Lagrangian particle placement fields are elements of this group.

We let

$$
\mathfrak{X}^{s}=\left\{u \in H^{s}(\Omega)^{3} \mid \operatorname{div} u=0, u \cdot n=0 \text { on } \partial \Omega\right\}
$$

denote the space of divergence-free velocity fields parallel to the boundary, which is, in a certain precise sense, the Lie algebra of $\mathcal{D}_{\mu}^{s}$.

For a given time interval $I=[0, T]$, let $\mathcal{C}=C^{\infty}\left(I, \mathcal{D}_{\mu}^{s}\right)$. Using Euler-Poincaré theory, it is easy to verify that the Euler equations arise from an application of Hamilton's principle to the kinetic energy action function. We shall recall directly how this goes: The action function is the map $S: \mathcal{C} \rightarrow \mathbb{R}$ given by

$$
\begin{aligned}
S(\eta(t, x)) & =\frac{1}{2} \int_{0}^{T} \int_{\Omega}|\dot{\eta}(t, x)|^{2} d x d t, \\
& =\frac{1}{2} \int_{0}^{T} \int_{\Omega}|u(t, x)|^{2} d x d t,
\end{aligned}
$$


where $\dot{\eta}(x, t)=\frac{\partial}{\partial t} \eta(x, t)$, and where the second equality follows from the fundamental relation between the Lagrangian velocity $\dot{\eta}$ and the Eulerian velocity $u$,

$$
u(t, \eta(t, x))=\dot{\eta}(t, x)
$$

the fact that Det $D \eta=1$, and the change-of-variables formula. We take the first variation of (10) and integrate by parts to obtain

$$
0=\delta S=-\int_{0}^{T} \int_{\Omega} \ddot{\eta}(t, x) \cdot \delta \eta d x d t .
$$

From (11), $\ddot{\eta}(t, x)=\partial_{t} u(t, \eta(t, x))+(u(t, \eta(t, x)) \cdot \nabla) u(t, \eta(t, x))$. Again using the change-of-variables formula, we see that

$$
0=\delta S=-\int_{0}^{T} \int_{\Omega}\left[\partial_{t} u(t, x)+(u(t, x) \cdot \nabla) u(t, x)\right] \cdot w(t, x) d x d t,
$$

where the vector field $w(t, x)$, given by $w(t, \eta(t, x)):=\delta \eta(t, x)$, is an arbitrary divergence-free variation (since $\eta$ is volume-preserving). Thus $\partial_{t} u+(u \cdot \nabla) u$ is $L^{2}$ orthogonal to all divergence-free vector fields $w$, and must therefore be the gradient of some function, say $p$. In other words, $u$ is a solution of the Euler equations

$$
\partial_{t} u+(u \cdot \nabla) u=-\operatorname{grad} p, \operatorname{div} u=0,
$$

or equivalently, in Lagrangian coordinates,

$$
\ddot{\eta}(t, x)=-\operatorname{grad} p(t, \eta(t, x)), \quad \text { Det } D \eta=1 .
$$

Remark 3. There is a very nice stochastic approach for the derivation of the NavierStokes equations in which the dissipative term can be obtained using a stochastic interpretation of the Lagrangian flow map. As can be seen from [4] and [20], by allowing the Lagrangian trajectory to have a superposed random walk, the diffusion term naturally arises. From the point of view of stochastic ordinary differential equations, the deterministic time derivatives are replaced with backward-in-time stochastic mean derivatives. By the Ito formula, the diffusion term naturally arises.

\section{Lagrangian averaging}

Initial Data and Averaging. Let $S$ denote the unit sphere in $\mathfrak{X}^{s}$. For $u_{0} \in \mathfrak{X}^{s}$, let $u(t, x)$ denote the solution of the Euler equations in $\mathfrak{X}^{s}$ with $u(0, \cdot)=u_{0}$. Let $u^{\varepsilon}(t, x)$ denote the solution of the Euler equations with initial data $u_{0}^{\varepsilon}$, where

$$
u_{0}^{\varepsilon}=u_{0}+\varepsilon w, \quad w \in S, \quad \varepsilon \in[0, \alpha]
$$

for a small parameter $\alpha>0$. Of course, $u^{\varepsilon}(t, x)$ depends on $w$ as well, but we suppress that for notational simplicity. By uniqueness of solutions, it follows that $u^{0}(t, x)=u(t, x)$. See Fig. 1. 


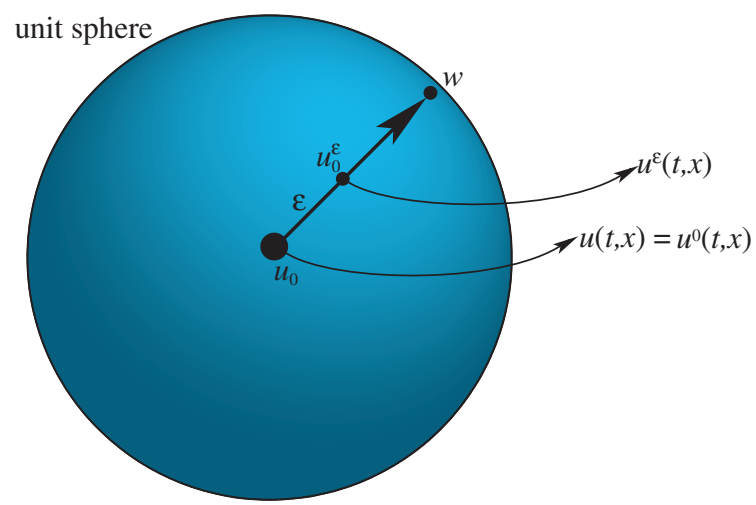

Fig. 1. Notation for the derivation of the averaged Euler equations.

We let $m$ denote a chosen measure on the unit sphere $S$ in $\mathfrak{X}^{s}$, we denote $u_{0}+S$ by $S_{u_{0}}$, and define the average of vector-valued functions $f(\varepsilon, w)$ on $[0, \alpha] \times S_{u_{0}}$ by

$$
\langle f\rangle:=\frac{\bar{t}}{\alpha} \int_{0}^{\alpha} \int_{S_{u_{0}}} f(\varepsilon, w) m(w) d \varepsilon,
$$

where $\bar{t}$ is a characteristic unit of time ${ }^{3}$. This will be our chosen ensemble averaging operation; namely, for each time $t$, we shall view $u^{\varepsilon}(t, \cdot ; w)$ as a vector-valued function defined on the ball $[0, \alpha] \times S_{u_{0}}$ and taking values in $\mathfrak{X}^{s}$. We shall also insist that our measure be invariant to the solution operator, so that for each $t$,

$$
\left\langle u^{\varepsilon}(t, x ; w)\right\rangle=u(t, x) .
$$

Thus, by invariance of the measure, our reference solution $u(t, x)$ is the mean of the ensemble of trajectories emanating from the cloud of initial data in the ball $[0, \alpha] \times S_{u_{0}}$. As such, we may perform a perturbation analysis in which we expand each trajectory $u^{\varepsilon}$ about the mean of the ensemble of trajectories $u$.

Flows and Fluctuations. Let $\eta$ be the Lagrangian flow of $u$ which solves

$$
\begin{aligned}
\dot{\eta}(t, x) & =u(t, \eta(t, x)), \\
\eta(0, x) & =x,
\end{aligned}
$$

and let

$$
\begin{aligned}
\dot{\eta}^{\varepsilon}(t, x) & =u^{\varepsilon}\left(t, \eta^{\varepsilon}(t, x)\right), \\
\eta^{\varepsilon}(0, x) & =\xi_{0}^{\varepsilon}(x)
\end{aligned}
$$

\footnotetext{
3 At this stage, $\alpha$ has units of velocity; later, we will make the change $\alpha \mapsto \alpha \bar{t} / 3$ to ensure that $\alpha$ ultimately has units of length.
} 
where for each $\varepsilon \in[0, \alpha], \eta^{\varepsilon}(t, x)$ is the flow of $u^{\varepsilon}(t, x)$ and where $\xi_{0}^{\varepsilon} \in \mathcal{D}_{\mu}^{s}$ is a near-identity volume-preserving diffeomorphism of $\Omega$ with $\xi_{0}^{\varepsilon=0}$ equal to the identity mapping; i.e., $\xi_{0}^{\varepsilon=0}(t, x)=x$.

We define the Lagrangian fluctuation volume-preserving diffeomorphism $\xi^{\varepsilon}$ by

$$
\xi^{\varepsilon}(t, y):=\eta^{\varepsilon}\left(t, \eta^{-1}(t, y)\right),
$$

where the inverse is taken with $t$ fixed; we also write this relation for short as $\xi^{\varepsilon}=\eta^{\varepsilon} \circ \eta^{-1}$. It can be equivalently written as

$$
\eta^{\varepsilon}(t, x)=\xi^{\varepsilon}(t, \eta(t, x)) .
$$

Note that $\xi_{0}^{\varepsilon}(x)=\xi^{\varepsilon}(0, x)$ since $\eta$ is the identity at $t=0$. See Fig. 2 .

In the present derivation, the decomposition (13) plays the role of the additive Reynolds decomposition (2) for vector fields. This decomposition is intrinsic, and uses the group structure of volume-preserving maps. It is reminiscent of (but is not the same as) the decomposition used by ANDREWS \& MCINTYRE [1] in their well-known GLM theory, which is given by equation (2.2) in [1] as (in our notation) $\eta^{\varepsilon}=\eta+\xi^{\varepsilon}$ and is made along a given particle trajectory, but not on the entire flow map.

The mapping $\eta$ represents the flow of the exact reference solution $u$ of the Euler equations, while $\xi^{\varepsilon}$ represents the fluctuations of the particles for a nearby solution of the Euler equations about the flow of this reference solution.

Expand the velocity field $u^{\varepsilon}$ about $\varepsilon=0$ as

$$
u^{\varepsilon}=u+\varepsilon u^{\prime}+\frac{1}{2} \varepsilon^{2} u^{\prime \prime}+O\left(\varepsilon^{3}\right),
$$

where

$$
u^{\prime}(t, y)=\left.\frac{d}{d \varepsilon}\right|_{\varepsilon=0} u^{\varepsilon}(t, y)
$$

and

$$
u^{\prime \prime}(t, y)=\left.\frac{d^{2}}{d^{2} \varepsilon}\right|_{\varepsilon=0} u^{\varepsilon}(t, y)
$$

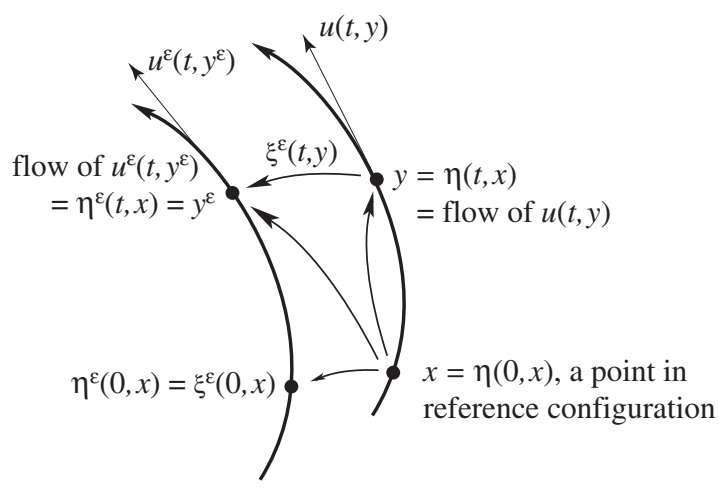

Fig. 2. Flow of the reference solution of the Euler equations and nearby flows. 
From our definition (12), $u$ is the mean velocity field, and to first order in $\varepsilon, u^{\prime}$ denotes the fluctuating component.

We will also need the derivatives of the fluctuation diffeomorphism $\xi^{\varepsilon}$ with respect to the perturbation parameter $\varepsilon$. The Lagrangian counterpart to $u^{\prime}$ is the divergence-free vector

$$
\xi^{\prime}(t, x)=\left.\frac{d}{d \varepsilon}\right|_{\varepsilon=0} \xi^{\varepsilon}(t, x),
$$

and the Lagrangian counterpart of $u^{\prime \prime}$ is

$$
\xi^{\prime \prime}(t, x)=\left.\frac{d^{2}}{d^{2} \varepsilon}\right|_{\varepsilon=0} \xi^{\varepsilon}(t, x) .
$$

Thus, the vector field $\xi^{\prime}$ is, to first order in $\varepsilon$, the Lagrangian fluctuation field.

We note that the vector fields $u^{\prime}(t, x), u^{\prime \prime}(t, x), \xi^{\prime}(t, x)$, and $\xi^{\prime \prime}(t, x)$ are functions of $w$ on the sphere $S_{u_{0}}$, but do not depend on $\varepsilon$ in the interval $[0, \alpha]$. For each of these fields, the averaging is simply over $S_{u_{0}}$.

Basic Derivative Relations. By differentiating the decomposition (14) with respect to both $t$ and $\varepsilon$, and evaluating at a general $t$, but $\varepsilon=0$, we obtain the relations

$$
\begin{aligned}
u^{\prime} & =\partial_{t} \xi^{\prime}+(u \cdot \nabla) \xi^{\prime}-\left(\xi^{\prime} \cdot \nabla\right) u \\
u^{\prime \prime} & =\partial_{t} \xi^{\prime \prime}+(u \cdot \nabla) \xi^{\prime \prime}-\left(\xi^{\prime \prime} \cdot \nabla\right) u-2\left(\xi^{\prime} \cdot \nabla\right) u^{\prime}-\nabla \nabla u\left(\xi^{\prime}, \xi^{\prime}\right),
\end{aligned}
$$

where, in coordinates,

$$
\nabla \nabla u\left(\xi^{\prime}, \xi^{\prime}\right)=u_{, j k}^{i} \xi^{\prime j} \xi^{\prime k} .
$$

The relation (15a) is the same as the relation between Eulerian and Lagrangian variations that is used in Euler-Poincaré reduction, and in particular, for the variational formulation of the Euler equations (see our earlier derivation of the Euler equations or, for example, [15]). It follows that

$$
\begin{aligned}
u^{\varepsilon}= & u+\varepsilon\left[\partial_{t} \xi^{\prime}+(u \cdot \nabla) \xi^{\prime}-\left(\xi^{\prime} \cdot \nabla\right) u\right] \\
& +\frac{\varepsilon^{2}}{2}\left[\partial_{t} \xi^{\prime \prime}+(u \cdot \nabla) \xi^{\prime \prime}-\left(\xi^{\prime \prime} \cdot \nabla\right) u-2\left(\xi^{\prime} \cdot \nabla\right) u^{\prime}-\nabla \nabla u\left(\xi^{\prime}, \xi^{\prime}\right)\right]+O\left(\varepsilon^{3}\right) .
\end{aligned}
$$

The Taylor Hypotheses. We are going to ensemble average over all possible solutions $u^{\varepsilon}$, but instead of substituting (16) into the Euler or Navier-Stokes equation and then averaging as in the Reynolds procedure, we shall instead average the action function as

$$
\langle S\rangle=\left\langle\frac{1}{2} \int_{0}^{T} \int_{\Omega}\left|u^{\varepsilon}\right|^{2} d x d t\right\rangle .
$$

The turbulence closure problem in our context then amounts to specifying the Lagrangian fluctuations $\xi^{\prime}$ and $\xi^{\prime \prime}$ as functions of the mean velocity $u$. To do so, we shall invoke the classical Frozen Turbulence Hypothesis introduced by TAYLOR 
in [25], and widely used by experimentalists in turbulence for the purpose of computing spatial derivatives from measurements that naturally yield only the temporal derivatives.

In its classical form, the streamwise scalar component $\zeta$ of the fluctuation is considered frozen over the time scale of the temporal derivative, giving

$$
\frac{\partial}{\partial t} \zeta+U \frac{\partial}{\partial x} \zeta=0
$$

where $U$ is the local mean velocity along the streamwise direction, which is here denoted by $x$. This is simply stating that the scalar fluctuation $\zeta$ is frozen into the mean flow. While the classical Taylor hypothesis deals with scalar fluctuations, in order to close our system, we must generalize his hypothesis to fluctuations which are divergence-free vector fields.

Definition 1. The generalized Taylor hypotheses to order $O\left(\varepsilon^{2}\right)$ are given as follows:

$$
\begin{gathered}
\partial_{t} \xi^{\prime}+(u \cdot \nabla) \xi^{\prime}-\left(\xi^{\prime} \cdot \nabla\right) u=0, \\
\frac{D}{d t}\left\langle\xi^{\prime \prime}\right\rangle \perp u
\end{gathered}
$$

where the orthogonality is taken in $L^{2}$ and, as usual,

$$
\frac{D}{d t} v=\partial_{t} v+(u \cdot \nabla) v .
$$

Equation (18a) states that the Lagrangian fluctuation $\xi^{\prime}$ is Lie advected or frozen into the mean flow as a divergence-free vector field, i.e., $\partial_{t} \xi^{\prime}+\mathfrak{f}_{u} \xi^{\prime}=0$. Note that this equation automatically preserves the divergence-free nature of $\xi^{\prime}$.

The initial data for (18) is given by $\xi^{\prime}(0, x)=\left.(d / d \varepsilon)\right|_{\varepsilon=0} \xi_{0}^{\varepsilon}$ and $\left\langle\xi^{\prime \prime}(0, x)\right\rangle=$ $\left\langle\left.\left(d^{2} / d \varepsilon^{2}\right)\right|_{\varepsilon=0} \xi_{0}^{\varepsilon}\right\rangle$. Notice that since the family of near-identity transformations $\xi_{0}^{\varepsilon}$ are volume-preserving, $\xi^{\prime}(t=0, \cdot)$ is divergence-free. It follows from the evolution equation (18a), that $\xi^{\prime}(t, \cdot)$ remains divergence-free. This observation is consistent with a fact mentioned before: for fixed $t, \xi^{\prime}$ is the derivative of the curve $\varepsilon \mapsto \xi^{\varepsilon}$ through the identity in the volume-preserving diffeomorphism group, so it must be divergence-free. Thus, the Taylor hypothesis is consistent with the divergence-free requirement.

Also note from (15a) that (18a) implies that $u^{\prime}=0$, so that $u^{\prime}$ is indeed a solution of the linearized Euler equations (as it must be), and the Eulerian fluctuations are relegated to second-order (and higher) effects in the perturbation parameter $\varepsilon$.

We claim that the Generalized Taylor Hypothesis produces a natural turbulence closure. To see this, we first substitute (18a) into (16) to get

$$
u^{\varepsilon}=u+\frac{\varepsilon^{2}}{2}\left[\partial_{t} \xi^{\prime \prime}+(u \cdot \nabla) \xi^{\prime \prime}-\left(\xi^{\prime \prime} \cdot \nabla\right) u-\nabla \nabla u\left(\xi^{\prime}, \xi^{\prime}\right)\right]+O\left(\varepsilon^{3}\right) .
$$

Next, define the Lagrangian covariance tensor $F$ by

$$
F=\left\langle\xi^{\prime} \otimes \xi^{\prime}\right\rangle
$$


substituting (19) into (17), using (18b), and using the dimensionally correct rescaling $\alpha \mapsto \alpha \bar{t} / 3$, we find that

$$
\langle S\rangle=\frac{1}{2} \int_{0}^{T} \int_{\Omega}\left\{|u|^{2}-\alpha^{2}\left[(u \cdot \nabla)\left\langle\xi^{\prime \prime}\right\rangle+\nabla \nabla u: F\right] \cdot u+O\left(\alpha^{3}\right)\right\} d x d t .
$$

Next, we truncate the averaged action to $O\left(\alpha^{2}\right)$ and call it $S_{\alpha}$, and search for a critical point, say $u_{\alpha}$, of

$$
S_{\alpha}=\frac{1}{2} \int_{0}^{T} \int_{\Omega}\left\{\left|u_{\alpha}\right|^{2}-\alpha^{2}\left[\left(u_{\alpha} \cdot \nabla\right)\left\langle\xi^{\prime \prime}\right\rangle+\nabla \nabla u_{\alpha}: F\right] \cdot u_{\alpha}\right\} d x d t .
$$

For notational convenience, we shall denote $u_{\alpha}$ simply as $u$.

Remark 4. In truncating the averaged action to $O\left(\alpha^{2}\right)$, we constrain the Lagrangian dynamics to the subgroup $\mathcal{D}_{\mu, D}^{s}$ of $\mathcal{D}_{\mu}^{s}$, consisting of those volume-preserving diffeomorphisms in $\mathcal{D}_{\mu}^{s}$ that leave the boundary $\partial \Omega$ fixed. In the Eulerian representation, this means that the vector field $u$ satisfies $u=0$ on $\partial \Omega$, and that its Lagrangian flow $\eta$ satisfies $\eta(t, x)=x$ for $x \in \partial \Omega$.

Remark 5. The subgroup $\mathcal{D}_{\mu, D}^{s}$ is also the appropriate configuration space for the Lagrangian fluctuations. This follows from the fact that in each "fluid experiment" (each solution of the Navier-Stokes equations), the turbulent velocity field must vanish along the boundary, which implies that the fluid particles cannot move along $\partial \Omega$. As such, the volume-preserving diffeomorphism $\xi^{\varepsilon}$ must satisfy $\xi^{\varepsilon}(t, x)=x$ for each $x \in \partial \Omega$ and each time $t$. Consequently, the Eulerian or spatial vector field $\xi^{\prime}$ must vanish on $\partial \Omega$. Since $F=\left\langle\xi^{\prime} \otimes \xi^{\prime}\right\rangle$, it is clear that the covariance degenerates to zero on the boundary. The equation (25) below shows that this condition is preserved by the transport equation that $F$ solves.

Using the boundary conditions discussed in Remark 4, we find that

$$
\begin{aligned}
S_{\alpha} & =\frac{1}{2} \int_{0}^{T} \int_{\Omega}\left\{|u|^{2}-\alpha^{2}\left[\operatorname{Div}(\nabla u \cdot F)-\nabla u \cdot\left(\left\langle\xi^{\prime \prime}\right\rangle-\operatorname{Div} F\right)\right] \cdot u\right\} d x d t, \\
& =\frac{1}{2} \int_{0}^{T} \int_{\Omega}\left\{|u|^{2}-\alpha^{2}\left[\operatorname{Div}(\nabla u \cdot F)-\frac{1}{2} \operatorname{grad}|u|^{2} \cdot\left(\left\langle\xi^{\prime \prime}\right\rangle-\operatorname{Div} F\right)\right]\right\} d x d t,
\end{aligned}
$$

where the first equality was obtained using the identity

$$
\operatorname{Div}(\nabla u \cdot F)-\nabla u \cdot \operatorname{Div} F=\nabla \nabla u \cdot F
$$

which is obvious from its coordinate expression

$$
\left(u_{, j}^{i} F^{j k}\right)_{, k}-u_{, j}^{i} F_{, k}^{j k}=u_{, j k}^{i} F^{j k},
$$

and integrating the term containing $\xi^{\prime \prime}$ by parts. The second equality was obtained by noting that $(\nabla u)^{T} \cdot u=\frac{1}{2} \operatorname{grad}|u|^{2}$.

To complete the closure of the dynamics, we must still remove the terms containing $\xi^{\prime \prime}$. This is accomplished with the following 
Lemma 1. For $u,\left\langle\xi^{\prime \prime}\right\rangle$, and $F$ sufficiently regular,

$$
\int_{\Omega} \operatorname{grad}|u|^{2} \cdot\left(\left\langle\xi^{\prime \prime}\right\rangle-\operatorname{Div} F\right) d x=0 .
$$

Proof. Notice that

$$
\xi^{\prime \prime}-\operatorname{Div}\left(\xi^{\prime} \otimes \xi^{\prime}\right)=\left.\frac{d}{d \varepsilon}\right|_{\varepsilon=0} \partial_{\varepsilon} \xi^{\varepsilon} \circ\left(\xi^{\varepsilon}\right)^{-1} .
$$

Since $\xi^{\varepsilon}$ is volume-preserving, the vector field $\partial_{\varepsilon} \xi^{\varepsilon} \circ\left(\xi^{\varepsilon}\right)^{-1}$ (this is the vector field $w^{\varepsilon}$ that generates $\xi^{\varepsilon}$ with $\varepsilon$ playing the role of time) is divergence-free, and hence so is its derivative with respect to $\varepsilon$ at $\varepsilon=0$. Since averaging and divergence commute, we see that

$$
\operatorname{div}\left(\left\langle\xi^{\prime \prime}\right\rangle-\operatorname{Div} F\right)=0
$$

The lemma follows from the fact that gradients are orthogonal to divergence-free vector fields.

Lemma 1 shows that

$$
\begin{aligned}
S_{\alpha} & =\frac{1}{2} \int_{0}^{T} \int_{\Omega}\left\{|u|^{2}-\alpha^{2} \operatorname{Div}(\nabla u \cdot F) \cdot u\right\} d x d t \\
& =\frac{1}{2} \int_{0}^{T} \int_{\Omega}\left\{|u|^{2}+\alpha^{2} \operatorname{Trace}(\nabla u \cdot F \cdot \nabla u)\right\} d x d t,
\end{aligned}
$$

where the second equality follows from integration by parts.

Remark 6. Note well that our derivation of the averaged action function $S_{\alpha}$ includes all terms up through order $\alpha^{2}$. This was indeed not the case in earlier derivations. Also note, that while the derivation we have presented above is a generalization (and refinement) of the method we used in [16] for the isotropic equations, by setting $F$ equal to a constant multiple of the identity matrix in (22), and taking into account the null Lagrangian, we recover the averaged action function producing the isotropic model, namely $\frac{1}{2} \int_{0}^{T} \int_{\Omega}\left\{|u|^{2}+\alpha^{2} \mid\right.$ Def $\left.\left.u\right|^{2}\right\} d x d t$. Note also, however, that in making the isotropy assumption, we have violated the fundamental fact that $F$ must vanish along $\partial \Omega$; for this reason, we believe the isotropic theory produces a good model of turbulence only on domains without boundary, such as the periodic-box.

Evolution of the Covariance Tensor. We will determine the equations for $u$ by appealing to Hamilton's principal for the truncated averaged action function $S_{\alpha}$, but first we determine the dynamics of $F$.

Lemma 2. The Lagrangian covariance tensor satisfies the equation

$$
\partial_{t} F+\nabla F \cdot u=\nabla u \cdot F+[\nabla u \cdot F]^{T},
$$


that is, the Lie transport equation

$$
\partial_{t} F+£_{u} F=0 .
$$

With $\eta(t, x)$ denoting the mean Lagrangian flow, the formula

$$
F(t, \eta(t, x))=[D \eta(t, x)] \cdot F_{0}(x) \cdot D \eta(t, x)^{T},
$$

where $F_{0}(x)=\left\langle\xi^{\prime}(0, x) \otimes \xi^{\prime}(0, x)\right\rangle$, also holds. Finally, the quantity Det $F(t, x)$ is conserved.

Proof. For (23), use the chain rule on (18a) and average. Equation (25) is equivalent to equation (23), and this can be checked by taking the time derivative of (25). The fact that Det $F$ is conserved follows from (25) and the fact that Det $D \eta=1$.

Remark 7. Using (25), and the fact that

$$
\nabla u=\nabla \dot{\eta} \cdot D \eta
$$

together with the change-of-variables formula, the averaged action function may be equivalently expressed as

$$
S_{\alpha}=\frac{1}{2} \int_{0}^{T} \int_{\Omega}\left[|\dot{\eta}|^{2}+\alpha^{2} \operatorname{Trace}\left(\nabla \dot{\eta} \cdot F_{0} \cdot \nabla \dot{\eta}\right)\right] d x d t
$$

Remark 8. Note that we require the Taylor hypothesis (18b) to hold as well as equation (21). Also note that the Taylor hypothesis (18b) holds whenever $\left\langle\xi^{\prime \prime}\right\rangle$ solves

$$
\partial_{t}\left\langle\xi^{\prime \prime}\right\rangle+(u \cdot \nabla)\left\langle\xi^{\prime \prime}\right\rangle=\nabla f
$$

for some $f$. If we choose $f$ by requiring

$$
\operatorname{div} \operatorname{Div}\left(£_{u} F+\left\langle\xi^{\prime \prime}\right\rangle \otimes u\right\rangle=\Delta f
$$

then there is no incompatibility between the Taylor hypothesis and (21).

Lemma 2 shows that the truncated averaged action function (22) has been closed; namely, $S_{\alpha}$ is only a function of the mean velocity $u$ and the covariance tensor $F$.

Applying Hamilton's principle directly to (22), just as we did in the previous section, or by making use of the semidirect Euler-Poincare theory (see [14]), we obtain the following evolution equation for $u$ :

$$
\partial_{t}\left(1-\alpha^{2} C\right) u+(u \cdot \nabla)\left(1-\alpha^{2} C\right) u=-\operatorname{grad} p, \quad \operatorname{div} u=0,
$$

where once again, $C u=\operatorname{Div}(\nabla u \cdot F)$. It is an easy computation to verify that the commutator

$$
\left[\partial_{t}+(u \cdot \nabla), C\right]=0,
$$

so that we obtain the inviscid form of the anisotropic LANS- $\alpha$ equations given in equations (6). 
Remark 9. Alternatively, we may compute the Euler-Lagrange equations by applying Hamilton's principle to the averaged action function (26); we obtain the anisotropic LAE- $\alpha$ equations in Lagrangian variables as

$$
\ddot{\eta}=-\left(1-\alpha^{2} C_{0}\right)^{-1} \operatorname{grad} p(t, \eta(t, x)),
$$

where $C_{0}(v)=\operatorname{Div}\left(\nabla v \cdot F_{0}\right)$.

Using the evolution equation (27) together with the procedure outlined in Remark 3, we obtain the diffusion term $v\left(1-\alpha^{2} C\right) \mathbb{P C} u$. We shall discuss the details of this stochastic approach in a future article.

For the isotropic theory which yields the isotropic LAE- $\alpha$ and LANS- $\alpha$ equations, we assume that the covariance tensor $F$ is a multiple of the identity matrix. In this case the averaged action function may be written as

$$
S_{\alpha}=\frac{1}{2} \int_{0}^{T} \int_{\Omega}\left[|u|^{2}+\alpha^{2}|\operatorname{Def} u|^{2}\right] d x d t ;
$$

application of Hamilton's principle then yields the isotropic equations.

\section{Well-posedness of the anisotropic equations}

We shall restrict our analysis to the three-dimensional periodic box, $\Omega=\mathbb{T}^{3}$, with $\mathbb{P}=P$. The case of a bounded domain with ellipticity degenerating on the boundary will be treated in [6] and [7], as well as the case where the projector $\mathbb{P}=\mathcal{P}_{F}^{\alpha}$. In fact, for wall-bounded flows, much more can be said about the role of the anisotropic equations; these issues are not treated here.

We shall use the notation $H_{\mathrm{per}}^{s}$ for $\left\{u \in\left[H^{s}\left(\mathbb{T}^{3}\right)\right]^{3} \mid \int_{\mathbb{T}^{3}} u(x) d x=0\right\}$. For $s \geqq 0$, set $H_{\text {div }}^{s}=\left\{u \in H_{\text {per }}^{s} \mid \operatorname{div} u=0\right\}$. For a linear operator $L: H^{s} \rightarrow H^{r}$, we denote

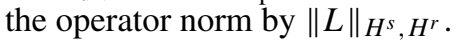

Definition 2. For any $f$ in the domain of $\left(1-\alpha^{2} C\right)$ and $F>0$, we set the Stokes projector $\mathcal{P}_{F}^{\alpha}(f)=w$, where $w$ is the solution of the generalized Stokes problem

$$
\begin{aligned}
\left(1-\alpha^{2} C\right) w+\operatorname{grad} p & =\left(1-\alpha^{2} C\right) f \\
\operatorname{div} w & =0 ;
\end{aligned}
$$

thus,

$$
\mathcal{P}_{F}^{\alpha}(f)=f-\left(1-\alpha^{2} C\right)^{-1} \operatorname{grad} p .
$$

Proposition 1. For $F>0$ and $F \in\left[H_{\mathrm{per}}^{r}\right]^{3 \times 3}, r>7 / 2, \mathcal{P}_{F}^{\alpha}: H_{\mathrm{per}}^{r} \rightarrow H_{\mathrm{div}}^{r}$ continuously.

Proof. Define the operator $\Lambda^{r}:=(1-\Delta)^{r / 2}$. For notational convenience, let $L=\left(1-\alpha^{2} C\right)$; we have

$$
L=\Lambda^{1-r} L \Lambda^{r-1}-\Lambda^{1-r}\left[L, \Lambda^{r-1}\right],
$$

so that the Stokes equation

$$
L u+\operatorname{grad} p=f, \quad \operatorname{div} u=0
$$


may be equivalently written as

$$
\Lambda^{1-r} L \Lambda^{r-1} u-\Lambda^{1-r}\left[L, \Lambda^{r-1}\right] u+\operatorname{grad} p=f, \quad \operatorname{div} u=0 .
$$

Letting $w=\Lambda^{r-1} u, \tilde{p}=\Lambda^{r-1} p$, and $g=\Lambda^{r-1} f$, (28) is equivalent to

$$
L w+\left[\Lambda^{r-1}, L\right] \Lambda^{1-r} w+\operatorname{grad} \tilde{p}=g, \quad \operatorname{div} w=0, \quad g \in H^{-1} .
$$

We claim that $\left[\Lambda^{r-1}, L\right] \Lambda^{1-r}: H^{1} \rightarrow L^{2}$ is bounded. This is an easy computation:

$$
\begin{aligned}
&\left\|\left[\Lambda^{r-1}, F^{j k} \frac{\partial^{2}}{\partial x^{j} \partial x^{k}}\right] \Lambda^{1-r} w\right\|_{L^{2}}+\left\|\left[\Lambda^{r-1}, F_{, k}^{j k} \frac{\partial}{\partial x^{j}}\right] \Lambda^{1-r} w\right\|_{L^{2}} \\
&=\left\|\left[\Lambda^{r-1}, F^{j k} \frac{\partial^{2}}{\partial x^{j} \partial x^{k}}\right] u\right\|_{L^{2}}+\left\|\left[\Lambda^{r-1}, F_{, k}^{j k} \frac{\partial}{\partial x^{j}}\right] u\right\|_{L^{2}} \\
& \leqq C\left(\left\|F^{j k}\right\|_{H^{r-1}}\left\|\partial_{j k}^{2} u^{i}\right\|_{L^{\infty}}+\left\|\nabla F^{j k}\right\|_{L^{\infty}}+\left\|\partial_{j k}^{2} u^{i}\right\|_{H^{r-2}}\right) \\
&+C\left(\|\operatorname{div} F\|_{H^{r-1}}\|\nabla u\|_{L^{\infty}}+\|\nabla \operatorname{div} F\|_{L^{\infty}}+\|\nabla u\|_{H^{r-2}}\right) \\
& \leqq C\left(\|F\|_{H^{r-1}}+\|\operatorname{div} F\|_{H^{r-1}}\right)\|w\|_{H^{1}},
\end{aligned}
$$

where we have used standard commutator estimates and the Sobolev embedding theorem for the last two inequalities. Thus,

$$
\left\|\left[\Lambda^{r-1}, L\right] \Lambda^{1-r}\right\|_{H^{1}, L^{2}} \leqq C\left(\|F\|_{H^{r-1}}+\|\operatorname{div} F\|_{H^{r-1}}\right) .
$$

Now, using Sobolev's embedding theorem, and our assumption that $F>0, F$ is elliptic and in $L^{\infty}$; a standard proof shows that the Stokes problem $L w+\operatorname{grad} \tilde{p}=g$, $\operatorname{div} w=0$ has a unique solution $w \in H_{\text {div }}^{1}$, and that $L: H_{\text {div }}^{1} \rightarrow H_{\text {per }}^{-1}$ has index 0 . By Rellich's theorem and the bound (30), the operator $\left[\Lambda^{r-1}, L\right] \Lambda^{1-r}: H_{\mathrm{div}}^{1} \rightarrow$ $H_{\text {per }}^{-1}$ is compact, and has trivial kernel. It follows that (29) has a unique solution $w \in H_{\text {div }}^{1}$ and thus (28) has a unique solution $u \in H_{\text {div }}^{r}$.

Remark 10. In the case where the coefficient $F$ (in the operator $C$ ) is in $W^{r-1, \infty}$, the above lemma follows from standard elliptic regularity results.

Theorem 1. For $s>7 / 2$, and $u_{0} \in H_{\text {div }}^{s}, F_{0} \in\left[H_{\text {per }}^{s}\left(\mathbb{T}^{3}\right)\right]^{3 \times 3}$, with $F_{0}>0$, there exists a unique solution $(u, F)$ with $u \in C^{0}\left([0, T] ; H_{\text {div }}^{s}\right) \cap L^{2}\left(0, T ; H_{\text {div }}^{s+1}\right)$ and $F \in C^{0}\left([0, T] ;\left[H_{\mathrm{per}}^{s}\left(\mathbb{T}^{3}\right)\right]^{3 \times 3}\right)$ to the anisotropic LANS- $\alpha$ equations $(6)$, where $T$ depends on the initial data.

Proof. The LANS- $\alpha$ equation (6a) may be written as

$$
\partial_{t} u+\mathcal{P}_{F}^{\alpha} \operatorname{Div}(u \otimes u)=v P \operatorname{div}[\nabla u \cdot F] .
$$

Let $u_{m}=P_{m} u$, where $P_{m}: H_{\text {per }}^{1} \rightarrow \operatorname{span}\left\{w^{1}, \ldots, w^{m}\right\}$ with $w^{j}$ denoting the $j$ th Fourier mode. We consider the Galerkin projection of (31) given by

$$
\begin{aligned}
\partial_{t} u_{m} & =-P_{m} \mathcal{P}_{F_{m}}^{\alpha} \operatorname{div}\left(u_{m} \otimes u_{m}\right)+v P_{m} \operatorname{div}\left[\left(\nabla u_{m} \cdot F_{m}\right) \nabla u_{m}\right] \\
u_{m}(0) & =P_{m} u_{0},
\end{aligned}
$$


where $F_{m}$ solves (23) with $u$ replaced by $u_{m}$. We drop the subscript $m$ from $F_{m}$ for notational convenience.

For each $m \in \mathbb{N}$, there is a smooth solution; in order to pass to the limit as $m \rightarrow \infty$ to produce a solution to (31), it suffices to obtain a priori estimates which do not depend on $m$.

Using (23) with $u$ replaced by $u_{m}$, it is easy to see that

$$
\begin{aligned}
+\|F(t, \cdot)\|_{H^{s}} & \leqq C\left\|F_{0}\right\|_{H^{s}} \exp \int_{0}^{t}\left\|u_{m}(s)\right\|_{H^{s+1}} d s \\
& \leqq C\left\|F_{0}\right\|_{H^{s}}\left(1+t\|u(t, \cdot)\|_{H^{s+1}}+O\left(t^{2}\right)\right) ;
\end{aligned}
$$

the last inequality makes use of a Taylor expansion about $t=0$.

Letting $\sigma$ denote a multi-index with $|\sigma| \leqq s$, we have

$$
\begin{aligned}
\frac{d}{d t}\left\|D^{\sigma} u_{m}\right\|_{L^{2}}^{2}= & -2\left(D^{\sigma} \mathcal{P}_{F}^{\alpha} \operatorname{div}\left(u_{m} \otimes u_{m}\right), D^{\sigma} u_{m}\right)_{L^{2}} \\
& -2 v\left(D^{\sigma}\left(F \cdot \nabla u_{m}\right), \nabla D^{\sigma} u_{m}\right)_{L^{2}} \\
\leqq & C\left\|\nabla u_{m} \cdot u_{m}\right\|_{H^{s}}\left\|u_{m}\right\|_{H^{s}}-2 v\left(F \cdot \nabla D^{\sigma} u_{m}, \nabla D^{\sigma} u_{m}\right)_{L^{2}} \\
& -2 v\left(\left[D^{\sigma}, u_{m}^{j}\right] \partial_{j} F, D^{\sigma} \nabla u_{m}\right)_{L^{2}},
\end{aligned}
$$

where we have used Proposition 1 with $s=r+1$ for the inequality. Since $F_{0}>0$, it follows from (25) that $F>0$, so that for some $\lambda>0$,

$$
-2 v\left(F \cdot \nabla D^{\sigma} u_{m}, \nabla D^{\sigma} u_{m}\right)_{L^{2}} \leqq-\lambda\left\|u_{m}\right\|_{H^{s+1}}^{2} .
$$

Using Young's inequality with $\varepsilon \in(0,1)$,

$$
\left\|\nabla u_{m} \cdot u_{m}\right\|_{H^{s}}\left\|u_{m}\right\|_{H^{s}} \leqq C(\varepsilon)\left\|u_{m}\right\|_{H^{s}}^{4}+\varepsilon\left\|u_{m}\right\|_{H^{s+1}}^{2},
$$

and so

$$
\begin{aligned}
2 v\left(\left[D^{\sigma}, u_{m}^{j}\right] \partial_{j} F, D^{\sigma} \nabla u_{m}\right)_{L^{2}} & \\
& \leqq\left\|\left[D^{\sigma}, u_{m}^{j}\right] \partial_{j} F\right\|_{L^{2}}\left\|u_{m}\right\|_{H^{s+1}} \\
& \leqq C\left(\left\|u_{m}\right\|_{H^{s}}\|\nabla F\|_{L^{\infty}}+\left\|u_{m}\right\|_{L^{\infty}}\|F\|_{H^{s}}\right)\left\|u_{m}\right\|_{H^{s+1}} \\
& \leqq C\left\|u_{m}\right\|_{H^{s}}\|F\|_{H^{s}}\left\|u_{m}\right\|_{H^{s+1}}, \\
& \leqq C\left\|F_{0}\right\|_{H^{s}}\left(1+O\left(t^{2}\right)\right)\|u\|_{H^{s}}\left\|u_{m}\right\|_{H^{s+1}}+t C\left\|F_{0}\right\|_{H^{s}}\left\|u_{m}\right\|_{H^{s}}\left\|u_{m}\right\|_{H^{s+1}}^{2} \\
& \leqq C(t, \varepsilon)\left\|F_{0}\right\|_{H^{s}}^{2}\left\|u_{m}\right\|_{H^{s}}^{2}+\varepsilon\left\|u_{m}\right\|_{H^{s+1}}^{2}+t C\left\|F_{0}\right\|_{H^{s}}\left\|u_{m}\right\|_{H^{s}}\left\|u_{m}\right\|_{H^{s+1}}^{2},
\end{aligned}
$$

where we have used a standard commutator estimate for the second inequality, the Sobolev embedding theorem for the third inequality, (33) for the fourth inequality, and Young's inequality for the last.

Suppose that $\left\|u_{m}\right\|_{H^{s}}<10\left\|u_{0}\right\|_{H^{s}}$; then by choosing $\varepsilon>0$ and $t>0$ sufficiently small so that

$$
10 \varepsilon+t C\left\|F_{0}\right\|_{H^{s}}\left\|u_{m}\right\|_{H^{s}}<\lambda,
$$

we have constants $\alpha>0$ and $\beta>0$ such that 


$$
\frac{d}{d t}\left\|u_{m}\right\|_{H^{s}}^{2} \leqq-\alpha\left\|u_{m}\right\|_{H^{s+1}}^{2}+\beta\left\|u_{m}\right\|_{H^{s}}^{4}+C(t, \varepsilon)\left\|F_{0}\right\|_{H^{s}}^{2} .
$$

It follows (by setting $\alpha=0$ ) that we may choose $t>0$ smaller if necessary so that $\left\|u_{m}\right\|$ is indeed smaller than $10\left\|u_{0}\right\|_{H^{s}}$ as was assumed.

From (34), we may conclude that for some $T>0, u_{m}$ is bounded in $L^{\infty}(0, T$; $\left.H_{\text {div }}^{s}\right) \cap W^{1, \infty}\left(0, T ; H_{\text {div }}^{s-2}\right) \cap L^{2}\left(0, T ; H_{\text {div }}^{s+1}\right)$, uniformly in $m$, and that a subsequence weakly converges to some $u$ in this space. By Ascoli's theorem, we have strong convergence in $C^{0}\left([0, T] ; H_{\mathrm{div}}^{S-2}\right)$, and hence by interpolation in $C^{\delta}([0, T]$; $\left.H_{\text {div }}^{s-2 \delta}\right)$, and by Sobolev's embedding theorem in $C^{0}\left([0, T] ; C_{\text {div }}^{2}\right)$ for $\delta$ taken sufficiently small. Thus, all the terms on the right-hand side of (32) converge strongly and $\partial_{t} u_{m}$ converges weakly. To see that $u \in C^{0}\left([0, T] ; H_{\text {div }}^{s}\right)$, it suffices to show that $\|u(t, \cdot)\|_{H^{s}}$ is continuous on $[0, T]$, but this follows from the above inequalities.

We next prove that the solution is unique. Assume that both $u_{1}$ and $u_{2}$ are solutions of (31), and that $F_{1}$ and $F_{2}$ are the respective solutions of (23). Denote by $\mathcal{P}_{1}^{\alpha}$ and $\mathcal{P}_{2}^{\alpha}$ the Stokes projectors associated with $F_{1}$ and $F_{2}$, respectively.

First, we claim that $\left\|\left(\mathcal{P}_{1}^{\alpha}-\mathcal{P}_{2}^{\alpha}\right) f\right\|_{H^{1}} \leqq C\left\|F_{1}-F_{2}\right\|_{H^{s}}$, whenever $\nabla f \in$ $L^{\infty}\left(\mathbb{T}^{n}\right)$. For $i=1,2$, let $g_{i}=\mathcal{P}_{i}^{\alpha} f$, so that $\left(1-\alpha^{2} C_{i}\right) g_{i}+\operatorname{grad} p_{i}=\left(1-\alpha^{2} C_{i}\right) f$. It follows that

$$
\left(1-\alpha^{2} C_{2}\right)\left(g_{1}-g_{2}\right)+\operatorname{grad}\left(p_{1}-p_{2}\right)=\alpha^{2} \operatorname{div}\left[\left(F_{1}-F_{2}\right) \cdot \nabla\left(g_{1}-f\right)\right] .
$$

Hence, for $v \in H_{\text {div }}^{1}$,

$$
\left(\left(1-\alpha^{2} C_{2}\right)\left(g_{1}-g_{2}\right), v\right)_{L^{2}}=\left(\left(F_{1}-F_{2}\right) \cdot \nabla\left(g_{1}-f\right), \nabla v\right)_{L^{2}} .
$$

Letting $v=g_{1}-g_{2}$, and using the coercivity of $F_{2}$, we get

$$
\lambda\left\|g_{1}-g_{2}\right\|_{H^{1}}^{2} \leqq C\left\|F_{1}-F_{2}\right\|_{L^{2}}\left\|g_{1}-g_{2}\right\|_{H^{1}},
$$

where the constant $C$ depends on $\|\nabla f\|_{L^{\infty}}$. Second, we claim that for $f \in H_{\text {per }}^{1}$, we have $\left\|\mathcal{P}_{i}^{\alpha} f\right\|_{H^{1}} \leqq C\|f\|_{H^{1}}$, where the constant $C$ depends on $\|F\|_{L^{\infty}}$. The variational form of the Stokes projector is given by

$$
\left(g_{i}, g_{i}\right)_{L^{2}}+\alpha^{2}\left(F_{i} \nabla g_{i}, \nabla g_{i}\right)_{L^{2}}=\left(f, g_{i}\right)_{L^{2}}+\alpha^{2}\left(F_{i} \nabla f, \nabla g_{i}\right)_{L^{2}},
$$

from which the result follows.

The evolution of the difference of $u_{1}$ and $u_{2}$ is given by

$$
\begin{aligned}
\partial_{t}\left(u_{1}-u_{2}\right)+ & \left(\mathcal{P}_{1}^{\alpha}-\mathcal{P}_{1}^{\alpha}\right) \nabla u_{1} \cdot u_{1}+\mathcal{P}_{2}^{\alpha} \nabla u_{1} \cdot\left(u_{1}-u_{2}\right) \mathcal{P}_{2}^{\alpha} \nabla\left(u_{1}-u_{2}\right) \cdot u_{2} \\
& =v \operatorname{div}\left[\left(F_{1}-F_{2}\right) \nabla u_{1}+F_{2} \nabla\left(u_{1}-u_{2}\right)\right],
\end{aligned}
$$

and the evolution for the difference of $F_{1}$ and $F_{2}$ is given by

$$
\begin{aligned}
& \partial_{t}\left(F_{1}-F_{2}\right)+\nabla\left(F_{1}-F_{2}\right) \cdot u_{1}+\nabla F_{2} \cdot\left(u_{1}-u_{2}\right) \\
& \quad=\left(F_{1}-F_{2}\right) \cdot \nabla u_{1}+F_{2} \cdot \nabla\left(u_{1}-u_{2}\right)+\nabla u_{1}^{T} \cdot\left(F_{1}-F_{2}\right)+\nabla\left(u_{1}-u_{2}\right)^{T} \cdot F_{2} .
\end{aligned}
$$

We set

$$
y(t)=\left\|u_{1}(t)-u_{2}(t)\right\|_{H^{1}}^{2}+\left\|F_{1}(t)-F_{2}(t)\right\|_{H^{1}}^{2} .
$$


Using the fact that

$$
\max _{t \in[0, T]}\left\|u_{i}(t, x)\right\|_{H^{s}}^{2}+\int_{0}^{T}\left\|u_{i}(t, x)\right\|_{H^{s+1}}^{2} d t \leqq K
$$

for some constant $K>0$ and $i=1,2$, and hence that $\left\|F_{i}\right\|_{H^{s}} \leq \tilde{K}$, a computation similar to that used for existence, using the above two claims, yields the differential inequality

$$
\frac{d}{d t} y(t) \leqq C(t) y(t), \quad C(t)=C\left(\left\|u_{i}\right\|_{H^{s}},\left\|F_{i}\right\|_{H^{s}}\right),
$$

from which uniqueness follows.

Acknowledgements. We thank MARCEL OLIVER for various helpful and important comments on the derivation, and DANIEL COUTAND for a number of helpful comments about the manuscript. We would also like to thank CIPRIAN FOIAS, DARRYL HOLM, and EDRISS TITI for many useful conversations on this topic. Finally, we would like to express our gratitude to the editor for his assistance in reading and correcting some of the finer points of the manuscript. JEM and SS were partially supported by the NSF-KDI grant ATM-98-73133. JEM also acknowledges the support of the California Institute of Technology. SS was also partially supported by NSF DMS-0105004 and the Alfred P. Sloan Foundation Research Fellowship.

\section{References}

1. ANDREWS, D., MCINTYRE, M.E.: An exact theory of nonlinear waves on a Lagrangianmean flow. J. of Fluid Mech., 89, 609-646 (1978)

2. ARNOLD, V.I.: Sur la géométrie differentielle des groupes de Lie de dimenson infinie et ses applications '91a l'hydrodynamique des fluids parfaits. Ann. Inst. Fourier, Grenoble, 16, 319-361 (1966)

3. Chen, S.Y., Foias, C., Holm, D.D., Olson, E.J., Titi, E.S., Wynne, S.: The Camassa-Holm equations as a closure model for turbulent channel and pipe flow. Phys. Rev. Lett., 81, 5338-5341 (1998)

4. ChORIN, A.J.: Numerical study of slightly viscous flow. J. Fluid Mech. 57, 785-796 (1973)

5. ChORIN, A.J., KAST, A.P., KuPFERMAN, R.: Unresolved computation and optimal predictions. Comm. Pure Appl. Math., 52, 1231-1254 (1999)

6. COUTAND, D., SHKOLLER, S.: Turbulent channel flow using the anisotropic Lagrangian averaged Navier-Stokes equations in weighted Sobolev spaces. (2002), preprint.

7. COUTAND, D., SHKOlLER, S.: The anisotropic Lagrangian averaged Navier-Stokes equations on bounded domains. (2002), in preparation.

8. EBIN, D.G., MARSDEN, J.E.: Groups of diffeomorphisms and the motion of an incompressible fluid. Ann. of Math. 92, 102-163 (1970)

9. Ercolani, N., Forest, M.G., Mclaughlin, D.W., Montgomery, R.: Hamiltonian structure of modulation equation for the sine-Gordon equation. Duke Math. J. 55, 949983 (1987)

10. FoIAS, C., Holm, D.D., TITI, E.S.: The three dimensional viscous Camassa-Holm equations and their relation to the Navier-Stokes equations and turbulence theory. Dynamics and Differential Equations 14, 1-36 (2002)

11. FRISCH, U.: Turbulence. The Legacy of A.N. Kolmogorov. Cambridge University Press, 1995

12. Holm, D.D.: Fluctuation effects on 3D Lagrangian mean and Eulerian mean fluid motion. Physica D, 133, 215-269 (1999) 
13. Holm, D.D., MARSDEN, J.E., RAtiU, T.S.: Euler-Poincaré models of ideal fluids with nonlinear dispersion. Phys. Rev. Lett. 349, 4173-4177 (1998)

14. Holm, D.D., MARSDEN, J.E., RATIU, T.S.: The Euler-Poincaré equations and semidirect products with applications to continuum theories. Adv. in Math. 137, 1-81 (1998)

15. MARSDEn, J.E., RATIU, T.S.: Introduction to Mechanics and Symmetry. Volume 17 of Texts in Applied Mathematics, 1994, Second Edition 1999, Springer-Verlag

16. Marsden, J.E., ShKoller, S.: Global well-posedness for the LANS- $\alpha$ Equation on bounded domains. Proc. Roy. Soc. London, 359, 1449-1468 (2001)

17. MELANDER, M., ZABUSKY, N.J., STYCZEK, A.S.: A moment model for vortex interactions of the 2D Euler equations I. Computational validation of an elliptical representation. J. Fluid Mech. 167, 95-115 (1986)

18. Mohseni, K., Kosovic, B., ShKoller, S., Marsden, J.E.: Numerical simulations of the Lagrangian averaged Navier-Stokes (LANS- $\alpha$ ) equations for homogeneous isotropic turbulence. Physics of Fluids, (2002), to appear; see http://www.math.ucdavis.edu/ shkoller/pub/pub.html.

19. Oliver, M., ShKoller, S.: The vortex blob method as a second-grade non-Newtonian fluid. Comm. Partial Differential Equations, 26, 294-314 (2001)

20. PeSKIN, C.: A randon-walk interpretation of the incompressible Navier-Stokes equations. Comm. Pure Appl. Math. 845, 845-852 (1985)

21. RIVLIN, R.S., ERICKSON, J.L.: Stress-deformation relations for isotropic materials. J. Rat. Mech. Anal., 4, 323-425 (1955)

22. SHKOLLER, S.: Geometry and curvature of diffeomorphism groups with $H^{1}$ metric and mean hydrodynamics. J. Funct. Anal. 160, 337-365 (1998)

23. SHKOLLER, S.: Analysis on groups of diffeomorphisms of manifolds with boundary and the averaged motion of a fluid. J. Differential Geometry, 55, 145-191 (2000)

24. Similon, P.L., KAUfMAN, A.N., Holm, D.D.: Oscillation center theory and pondermotive stabilization of the low-frequency plasma modes. Phys. Fluids 29, 1908-1922 (1986)

25. TAYLOR, G.I.: The spectrum of turbulence. Proc. R. Soc. London Ser. A, 164, 476-490 (1938)

26. Whitham, G.: Linear and Nonlinear Waves. Wiley-Interscience, 1974

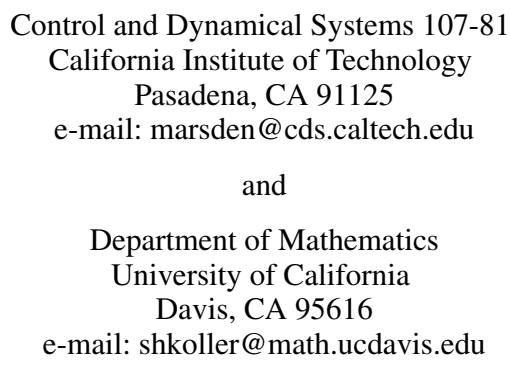

(Accepted September 2, 2002)

Published online November 26, 2002 - (C) Springer-Verlag (2002) 